\title{
On the Accuracy of X-ray Spectra Modeling of Inertial Confinement Fusion Plasmas
}

S.H. Glenzer, K.B. Fournier, B.A. Hammel, R.W. Lee, B.J. MacGowan and C.A. Back

This article was submitted to $12^{\text {th }}$ American Physical Society Topical Conference on Atomic Processes in Plasmas, Reno, NV, March 19-23, 2000

U.S. Department of Energy

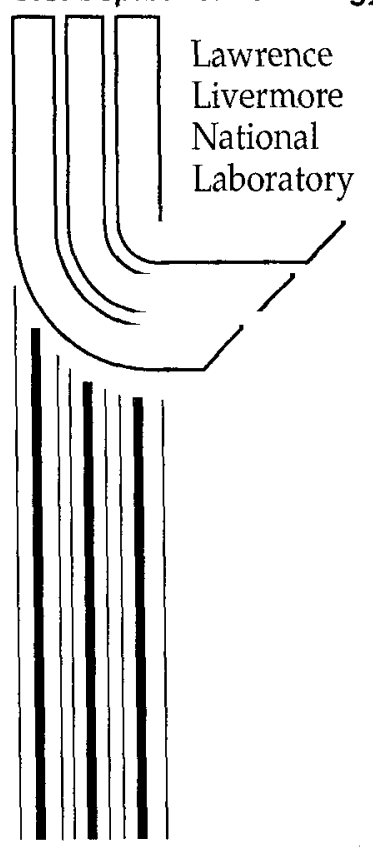

May 3, 2000 


\section{DISCLAIMER}

This document was prepared as an account of work sponsored by an agency of the United States Government. Neither the United States Government nor the University of California nor any of their employees, makes any warranty, express or implied, or assumes any legal liability or responsibility for the accuracy, completeness, or usefulness of any information, apparatus, product, or process disclosed, or represents that its use would not infringe privately owned rights. Reference herein to any specific commercial product, process, or service by trade name, trademark, manufacturer, or otherwise, does not necessarily constitute or imply its endorsement, recommendation, or favoring by the United States Government or the University of California. The views and opinions of authors expressed herein do not necessarily state or reflect those of the United States Government or the University of California, and shall not be used for advertising or product endorsement purposes.

This is a preprint of a paper intended for publication in a journal or proceedings. Since changes may be made before publication, this preprint is made available with the understanding that it will not be cited or reproduced without the permission of the author.

This report has been reproduced directly from the best available copy.

Available to DOE and DOE contractors from the Office of Scientific and Technical Information

P.O. Box 62, Oak Ridge, TN 37831

Prices available from (423) 576-8401

http://apollo.osti.gov/bridge/

Available to the public from the National Technical Information Service

U.S. Department of Commerce 5285 Port Royal Rd., Springfield, VA 22161 http://www.ntis.gov/

OR

Lawrence Livermore National Laboratory Technical Information Department's Digital Library http://www.llnl.gov/tid/Library.html 


\title{
On the Accuracy of X-ray Spectra Modeling of Inertial Confinement Fusion Plasmas
}

\author{
S. H. Glenzer, K. B. Fournier, B. A. Hammel, R. W. Lee, B. J. MacGowan, \\ and C. A. Back
}

Lawrence Livermore National Laboratory, P.O. Box 808, L-437, Livermore CA 94550

\section{INTRODUCTION}

We have tested $\mathrm{x}$-ray emission spectroscopic modeling of the helium-like and lithium-like argon emission in dense gas bag plasmas by comparing measured spectra with kinetics calculations using plasma parameters that have been independently and accurately measured with Thomson scattering. In particular, we have measured the line radiation in the wavelength region of the He-like $\mathrm{Ar} 1 \mathrm{~s}^{2}-1 \mathrm{~s} 3 \ell$ transition $(\mathrm{He}-\beta)$. This spectrum is of interest to diagnose gas targets, $\quad \mathrm{n}_{\mathrm{e}} \sim$ $10^{21} \mathrm{~cm}^{-3}(1,2,3)$, laser ablation plasmas, $n_{e} \sim 10^{22} \mathrm{~cm}^{-3}(4)$, and has previously been applied to diagnose electron densities and temperatures of inertial confinement fusion (ICF) capsule implosions, $\mathrm{n}_{\mathrm{e}} \sim 10^{24} \mathrm{~cm}^{-3}(5,6)$.

The ICF implosions produce plasma conditions of extremely high densities similar to those of stars and therefore require $\mathrm{x}$-ray emission or neutron diagnostics (7). The spectrum of the He- $\beta$ transition of Ar XVII together with its dielectronic satellites arising from the Li-like Ar states $1 s^{2} n \ell-1 \mathrm{~s} n \ell n^{\prime} \ell^{\prime}$, referred to below as the He- $\beta$ complex, has been found to be a valuable diagnostic of electron densities and temperatures. The He- $\beta$ line is Stark-broadened so that densities can be inferred from the width of the spectral line. Moreover, the upper states of the observable dielectronic satellites on the red wing of the $\mathrm{He}-\beta$ line are predominantly populated by dielectronic recombination so that their relative intensity is sensitive to the electron temperature $(8,9)$. Some of the higher-n satellite features overlap with the $\mathrm{He}-\beta$ transition and consequently need to be self-consistently included in the fit of the whole line shape with a Stark-broadening code coupled to a kinetics (collisionalradiative) model $(10,11)$. This procedure applies kinetics modeling to very high densities where the codes have not been tested against independent measurements. In this study, we perform critical comparisons of kinetics calculations with experimental data from well-characterized plasmas at the highest possible densities where independent optical diagnostics, i.e., Thomson scattering, can be used to measure the electron temperature $(12,13)$. This is a necessary first step toward a critical evaluation of the diagnostic procedures used at the highest measured plasma density of $n_{e}>10^{24} \mathrm{~cm}^{-3}$.

We have performed our experiments in well-characterized gas bag plasmas at densities $n_{e}=0.6 \times 10^{21} \mathrm{~cm}^{-3}$ and $n_{e}=1.1 \times 10^{21} \mathrm{~cm}^{-3}$. The densities of these gas bag plasmas are independently diagnosed with stimulated Raman scattering (1), and the 
electron temperatures are measured with temporally and spatially resolved Thomson scattering (13). The Thomson scattering measurements indicate that the gas bags are homogeneous with slowly increasing electron temperatures during the first $0.6 \mathrm{~ns}$ of the Ins long heater beam pulse. These data are also consistent with hydrodynamic LASNEX modeling (14) suggesting that gas bag plasmas are suitable sources to test our kinetics modeling capability.

To compare the experimental spectra with synthetic spectra, we employ the HULLAC suite of kinetics codes (15). We find for the two different electron densities that the kinetics modeling accurately predicts the intensity ratio of the Lilike dielectronic capture satellite transitions and of the He- $\beta$ transition (consisting of the sum of the resonance line, He- $\beta_{1}: 1 \mathrm{~s}^{2}{ }^{1} \mathrm{~S}_{0}-1 \mathrm{~s} 3 \mathrm{p}{ }^{1} \mathrm{P}^{0}{ }_{1}$, and the less intense intercombination line from the triplet to the singlet system of $\mathrm{He}$-like argon, $\mathrm{He}-\beta_{2}$ : $\left.1 \mathrm{~s}^{2} \mathrm{~S}_{0}-1 \mathrm{~s} 3 \mathrm{p}^{3} \mathrm{P}_{1}^{0}\right)$. On the other hand, spectral line emission originating from levels whose population is primarily determined by electron collisional processes shows discrepancies of up to a factor of two compared to the modeling. This is particularly observed for inner-shell excited satellite transitions that are populated from the lithium-like ground state. We have examined possible reasons for this discrepancy and found that a likely explanation might be errors of the calculated ionization balance between the helium- and lithium-like state.

In spite of these remaining discrepancies between calculated and measured inner shell satellite intensities, the fact that the strongest satellite features, i.e. the dielectronic capture satellites, are well modeled by the HULLAC code may affect the interpretation of ICF capsule implosions experiments. Our findings indicate that we should revisit the analysis of the higher density implosions to find if the kinetics modeling is consistent with the results obtained here. Preliminary calculations for implosion conditions show that various kinetics codes result in a factor of two different prediction for the ratio of the capture satellites to the He- $\beta$ transition. More analysis using line shape calculations will be required to investigate whether the previous interpretation of spectra from capsule implosion is affected (6). Moreover, having proven the technique to benchmark kinetics calculations in laserproduced plasma conditions, one can hope to extend this method to verify critically important aspects of indirectly driven capsule physics by testing the conditions created inside of ICF hohlraums.

\section{EXPERIMENT}

The experiments were performed with the Nova laser facility at the Lawrence Livermore National Laboratory (16). This Nd:glass laser was operating at 1.055 $\mu \mathrm{m}(1 \omega)$ and could be frequency converted to $3 \omega$ with energies of $\sim 30 \mathrm{~kJ}$. The application of these large laser energies has enabled us to produce large scale-length and extremely homogeneous plasmas $\left(\Delta \mathrm{T}_{\mathrm{e}} / \mathrm{T}_{\mathrm{e}}<20 \%\right)$. We used nine $\mathrm{f} / 4.3$ laser beams to illuminate gas bag targets from all sides. Gas bags consist of two $0.35 \mu \mathrm{m}$ thick polyimide $\left(\mathrm{C}_{14} \mathrm{H}_{6} \mathrm{O}_{4} \mathrm{~N}_{2}\right)$ membranes that are mounted on either side of a $0.4 \mathrm{~mm}$ thick aluminum washer with an inner diameter of $2.75 \mathrm{~mm}$ (Fig. 1). In this study, the membranes have been inflated with propane $\left(\mathrm{C}_{3} \mathrm{H}_{8}\right)$ or neopentane 


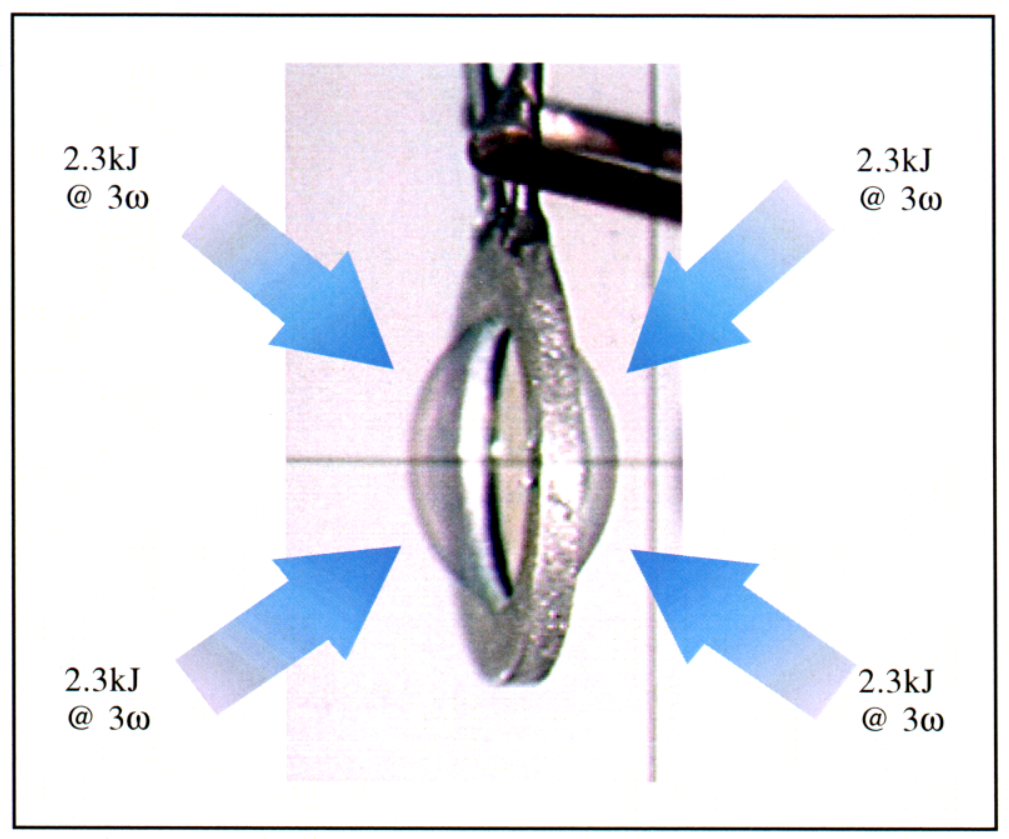

Figure 1. Gas bag target before it was pressurized with $\mathrm{C}_{3} \mathrm{H}_{8}$ or $\mathrm{C}_{5} \mathrm{H}_{12}$. The thickness and the inner diameter of the washer were $0.4 \mathrm{~mm}$ and $2.75 \mathrm{~mm}$, respectively. The gas bags were heated with nine $1 \mathrm{~ns}$-long heater beams of $2.3 \mathrm{~kJ}$ per beam at $3 \omega$.

$\left(\mathrm{C}_{5} \mathrm{H}_{12}\right)$ plus a small amount of argon $(1 \%$ by atomic number in each case) as spectroscopic test element. The concentration of $\mathrm{Ar}$ atoms of $1 \%$ was chosen to obtain optically thin conditions for the $\mathrm{Li}$ - and He-like $\mathrm{Ar}$ emission. The heater beams provided $2.3 \mathrm{~kJ}$ energy per beam at $3 \omega\left(\lambda_{0}=351 \mathrm{~nm}\right)$ in a $1 \mathrm{~ns}$-long square pulse. A diverging focus resulted in an intensity of $\mathrm{I}=10^{14} \mathrm{~W} \mathrm{~cm}^{-2}$ on target.

The X-ray spectra have been measured with a crystal spectrometer (17) coupled to a gated microchannelplate detector (MCP) (18). The x-ray emission is observed through a slit cut in a copper shield and mounted on the target at a distance of $8 \mathrm{~mm}$, effectively limiting the plasma size seen by the spectrometer. This slit allows a view through the gas bag center. We used a pentaerythritol (PET) crystal to spectrally disperse the plasma emission and detected the spectra with the MCP detector with a temporal resolution of $80 \mathrm{ps}$, a spatial resolution of $22 \mu \mathrm{m}$, and a spectral resolution of $\lambda / \Delta \lambda=800$. Examples of the spectra measured in this way are presented in the next section. The gated MCP detector was further employed for 2-D x-ray imaging of the gas bag emission with photon energies of $\mathrm{E}>2 \mathrm{keV}$. For this purpose, we used $10 \mu \mathrm{m}$ pinholes with a proper Be filter in front of the MCP camera. Figure 2 shows an example of three successive measurements. For early times $(t<0.3 \mathrm{~ns})$ during the 1ns-long heating pulse, the initial imprint of the heater beams can be identified. For $\mathrm{t}>0.4 \mathrm{~ns}$, these measurements indicate that the gas bag plasmas turn homogeneous.

For a more quantitative investigation of the plasma homogeneity, we measured the electron temperature at various distances from the target center with Thomson 


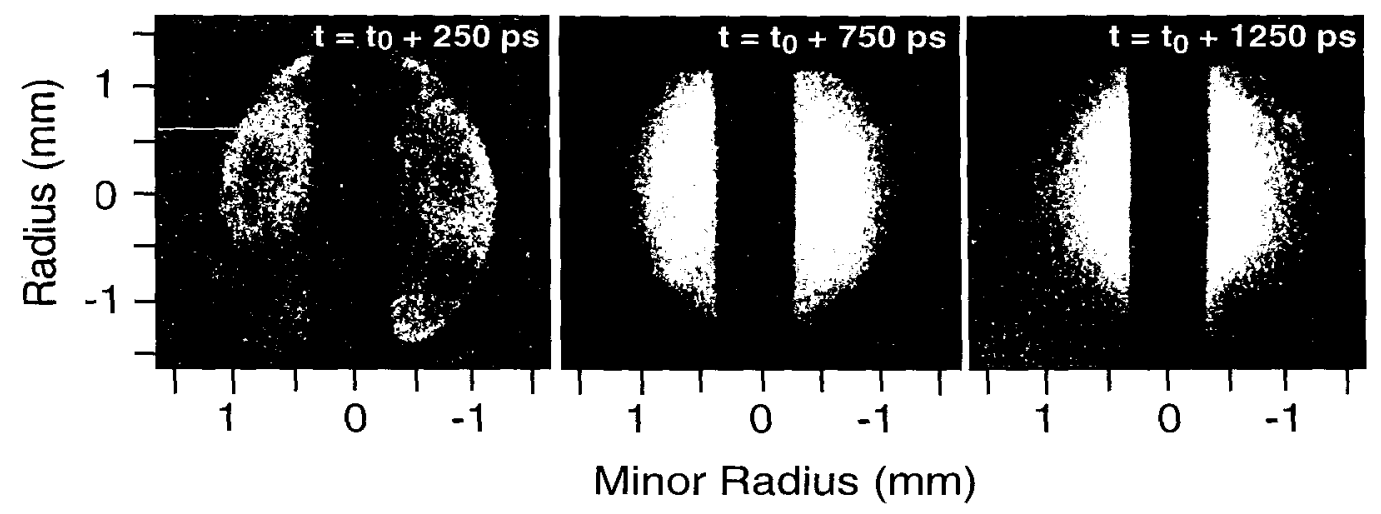

Figure 2. Temporally resolved two-dimensional x-ray images of the gas bag emission with energies $\mathrm{E}>2 \mathrm{keV}$. The gas bags show a homogeneous emission shortly after the beginning of the heater pulse (for $t>0.4 n s$ ).

scattering. A $50-\mathrm{J}, 4 \omega\left(\lambda_{0}=263 \mathrm{~nm}\right)$ probe beam has been used for the Thomson scattering experiments (13). A short-wavelength probe is required to characterize open geometry large-scale length ICF plasmas, because of strong laser light absorption, stray light, as well as stimulated Raman side scattering from the heater beams in the wavelength region around the longer Nd:glass wavelength harmonics: $2 \omega$ and $3 \omega$. The probe laser was focussed into the gas bag target to a spot of $60 \mu \mathrm{m}$ $x 120 \mu \mathrm{m}$ resulting in an intensity of $\mathrm{I} \sim 3 \times 10^{14} \mathrm{Wcm}^{-2}$ (at $\left.4 \omega\right)$. In separate experiments, we have shown that this probe does not influence the plasma as long as it is hot and heated by kJ-laser beams.

The Thomson scattered light has been imaged at an angle of $90^{\circ}$ with f/10-optics onto the entrance slit of a $1 \mathrm{~m}$ (SPEX) spectrometer. We employed an S-20 streak camera to record spectra with a temporal resolution of 50ps and a wavelength resolution of $0.05 \mathrm{~nm}$. The imaging setup resulted in a cylindrical scattering volume with a scale length of $\sim 100 \mu \mathrm{m}$ in all directions. The scattering volume is small compared to the size of the plasma. The choice of the probe laser wavelength of $263 \mathrm{~nm}$ and of the scattering angle of $90^{\circ}$ results in collective Thomson scattering from fluctuations characterized by wave numbers $\mathbf{k}$ such that the scattering parameter is $\alpha=1 / \mathrm{k} \lambda_{\mathrm{D}}>2$ for the gas bag electron densities and temperatures. The Thomson scattering spectra are dominated by the narrow ion feature which shows scattering resonances at the ion acoustic wave frequencies shifted from the incident probe laser frequency on either side on the frequency scale (red shift and blue shift for copropagating and counterpropagating waves along the scattering vector $\mathbf{k}$.

Figure 3 shows an example of Thomson scattering spectra at $t=0.35 n$ s and $t=$ $0.95 \mathrm{~ns}$ measured at a radius of $800 \mu \mathrm{m}$ from the gas bag center. The electron temperature can be inferred from the frequency separation of the two ion acoustic peaks. Each peak consists of two unresolved ion acoustic waves, one belonging to carbon (slow mode) and one belonging to hydrogen (fast mode) giving the ion temperature of the plasma from the relative damping of these waves. With increasing time, the ion acoustic peaks show increased separation and broadening 


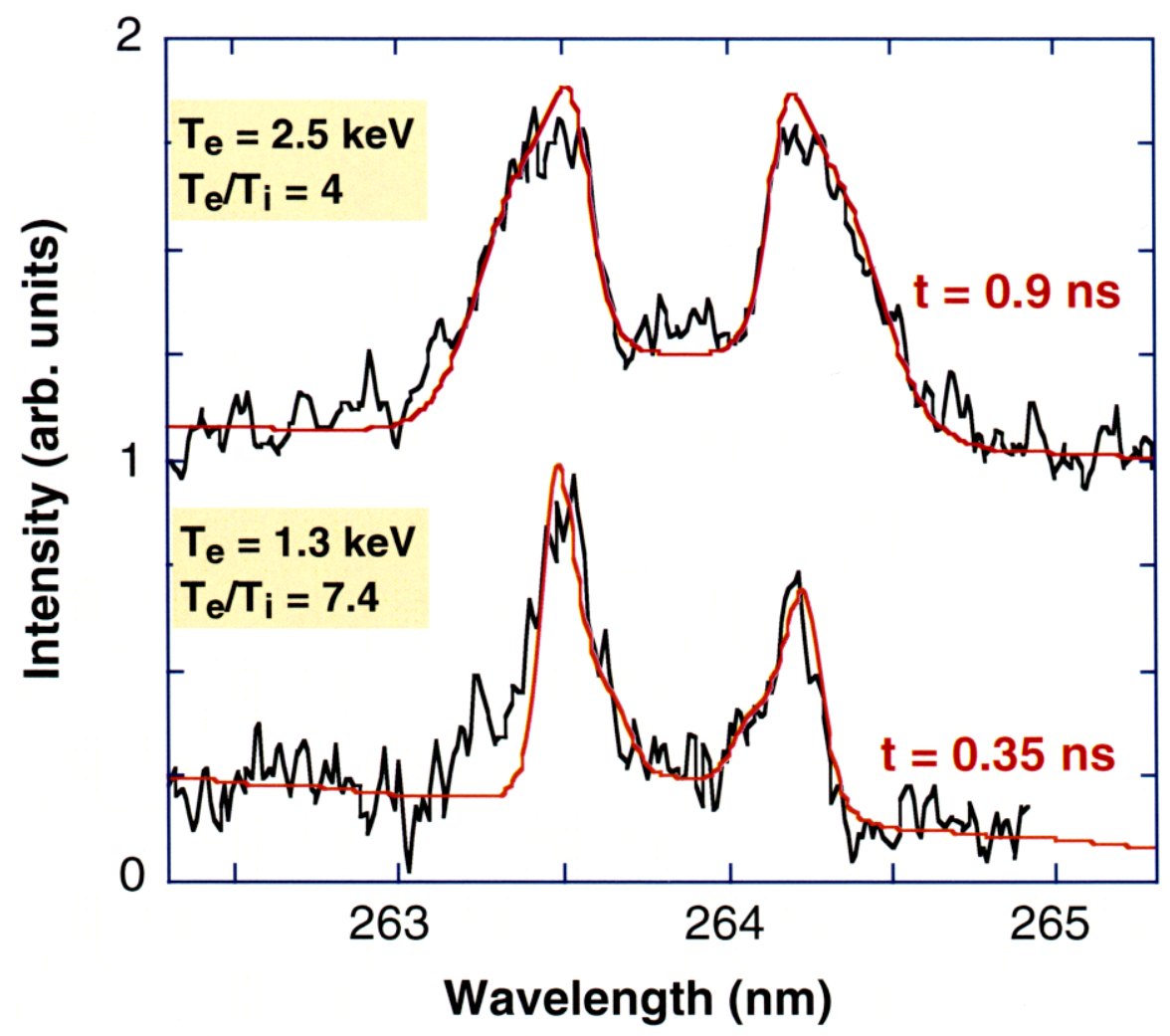

Figure 3. Experimental Thomson scattering spectra from a $\mathrm{C}_{5} \mathrm{H}_{12}$-filled gas bag measured from a radial distance of $0.8 \mathrm{~mm}$ from the gas bag center. The spectra show increasing electron temperatures and decreasing electron to ion temperature ratios. The parameters are inferred from the theoretical fits to the experimental data.

indicating increasing electron and ion temperatures during the heating of the gas bag plasma. To accurately infer temperatures from these spectra, we convolute the form factor $S(\mathbf{k}, \omega)$ for multi-ion species (19) with the experimental instrument function and fit the resulting profile to the data. Examples of these fits are also shown in Fig. 3. We obtain an error estimate for the electron temperature of $<10 \%$ from the fitting procedure by varying the calculated profile within the noise of the experimental data.

Figure 4 shows the electron temperature as function of the radius for two different times during the heating of the gas bag plasma. The experimental data show mutual agreement between the results from the temporally and spatially resolved Thomson scattering technique and with temporally and spatially resolved $\mathrm{x}$ ray spectroscopy using the intensity ratio of the He-like $\mathrm{Ar}_{1} \mathrm{~s}^{2}-1 \mathrm{~s} 2 \mathrm{l}(\mathrm{He}-\alpha)$ line to the lithium-like jk $\ell$ dielectronic satellites (2). These two techniques are compared together with hydrodynamic LASNEX and FCI2 simulations. The error bars for the spectroscopically derived temperatures are in the range of $15 \%-20 \%$ depending on the noise amplitude of the individual spectra at various times. The Thomson scattering data are accurate to within $10 \%$. We find that the results of the 


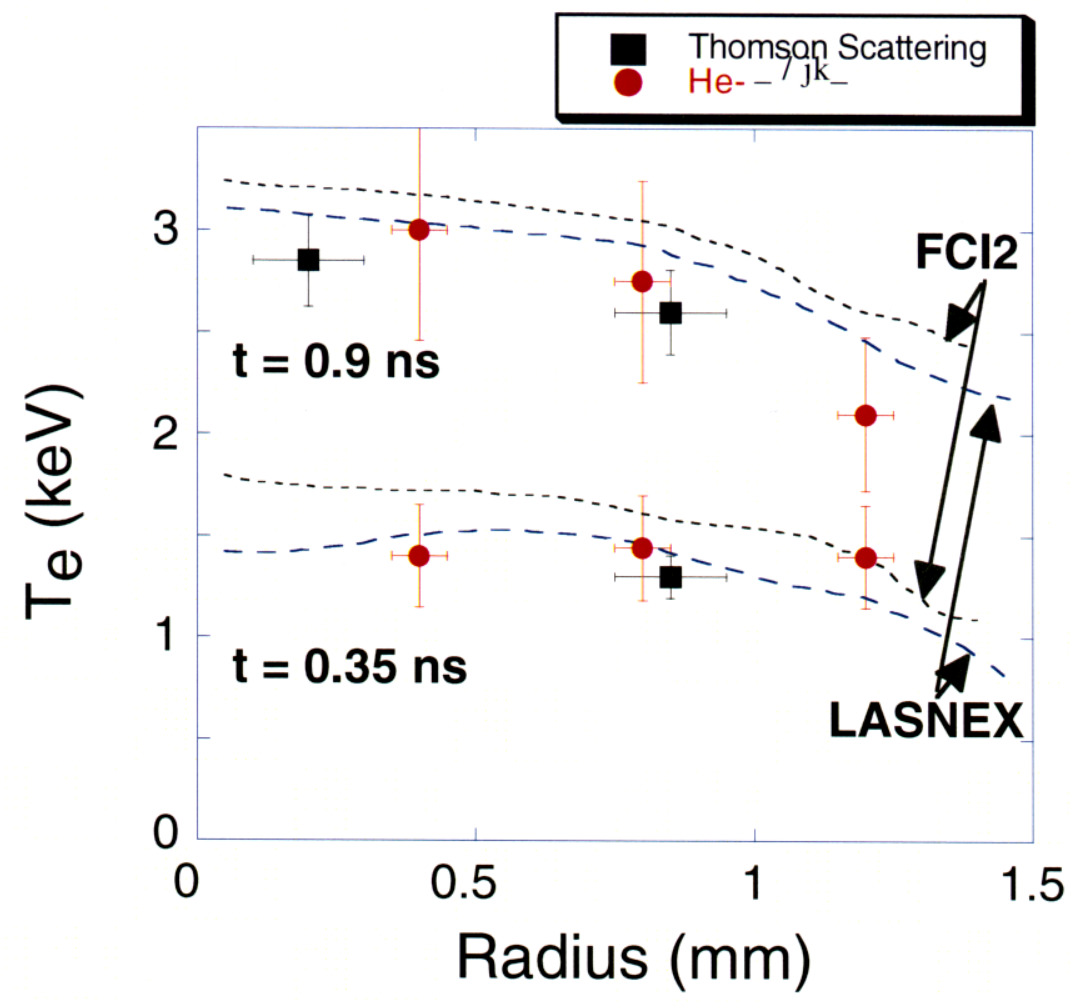

Figure 4. Experimental electron temperature data for various radial positions measured at $t=0.35 \mathrm{~ns}$ and at $\mathrm{t}=0.9 \mathrm{~ns}$. The temperatures from Thomson scattering show excellent agreement with the results from x-ray spectroscopy and are consistent with the hydrodynamic modeling using the codes LASNEX and FCI2. At $\mathrm{t}=0.35 \mathrm{~ns}$ the electron temperature profile is flat indicating the utility of gas bags for spectroscopic investigations.

simulations are close to the experimental data. For this comparison, we include the heater beam scattering losses by SBS and SRS. The results presented in Fig. 4 clearly show a homogeneous plasma at the time of the measurements of the He- $\beta$ transition plus satellites, i.e. $0.3 \mathrm{~ns}<\mathrm{t}<0.5 \mathrm{~ns}$, with $\Delta \mathrm{T}_{\mathrm{e}} / \mathrm{T}_{\mathrm{e}}<20 \%$. At the time close to peak temperature, i.e. $\mathrm{t}=0.9 \mathrm{~ns}$, we find a homogeneous center with a diameter of $2 \mathrm{~mm}, \Delta \mathrm{T}_{\mathrm{e}} / \mathrm{T}_{\mathrm{e}}<30 \%$

While the electron and ion temperatures in these gas bag plasmas are well known from the measurements described above, the electron density is principally known by the density of the gas fill. Measurements of the wavelength of the Raman scattered light which occurs at the frequency of the electron plasma/Langmuir wave give a value for the electron density which is consistent with the gas fill density (1). The line intensity ratio of the resonance and intercombination line of helium-like argon have also been shown to be in agreement with the expected densities (2). In summary, the detailed measurements and the general agreement with the simulations indicate that the plasma conditions in these gas bag targets are known with good accuracy so that it provides a spectroscopic source which is very suitable to test kinetics codes. 


\section{EXPERIMENTAL RESULTS AND DISCUSSION}

Figure 5 shows an example of a temporally resolved x-ray spectrum in the wavelength region $0.332 \mathrm{~nm}<\lambda<0.345 \mathrm{~nm}$ measured at $\mathrm{t}=0.35 \mathrm{~ns}$ from a gas bag that has been filled with $\mathrm{C}_{5} \mathrm{H}_{12}$ and $1 \% \mathrm{Ar}$. The data have been measured spatially resolved along the slit height and averaged over the region $r<1.2 \mathrm{~mm}$ to reduce noise. The averaging is justified because at the time of the measurements the gas bag plasma is homogeneous as shown above. In Fig. 5 we also show a synthetic spectrum calculated with the experimental resolution and with a higher resolution using the HULLAC suite of codes for the experimental plasma parameters: $\mathrm{T}_{\mathrm{e}}=1.3 \mathrm{keV}, \mathrm{n}_{\mathrm{e}}=1.1 \times 10^{21} \mathrm{~cm}^{-3}$.

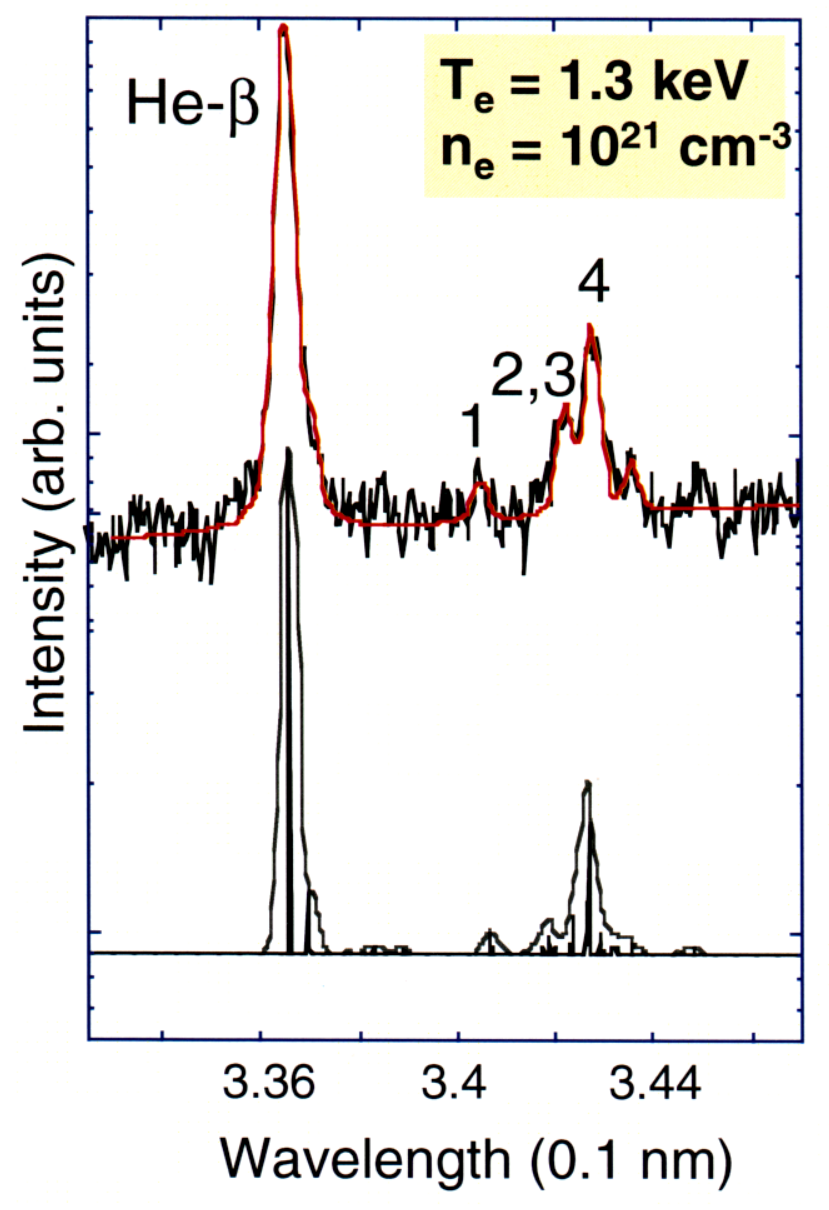

Figure 5. Experimental and synthetic spectra of the Ar He- $\beta$ complex.

The spectrum is dominated by the $1 \mathrm{~s}-3 \mathrm{p}$ resonance line of helium-like argon ArXVII at $\lambda=0.3364 \mathrm{~nm}$, He- $\beta$, the theoretical transition energy of which has been used to determine the absolute wavelength scale of Fig. 5. A number of spectral lines can be identified on the red wing of the He- $\beta$ transition. These are the intercombination line at $\lambda=0.337 \mathrm{~nm}$, dielectronic satellites with a $\mathrm{n}=3$ spectator electron at $\lambda=0.3381 \mathrm{~nm}$ and four dielectronic satellites with a $\mathrm{n}=2$ spectator 


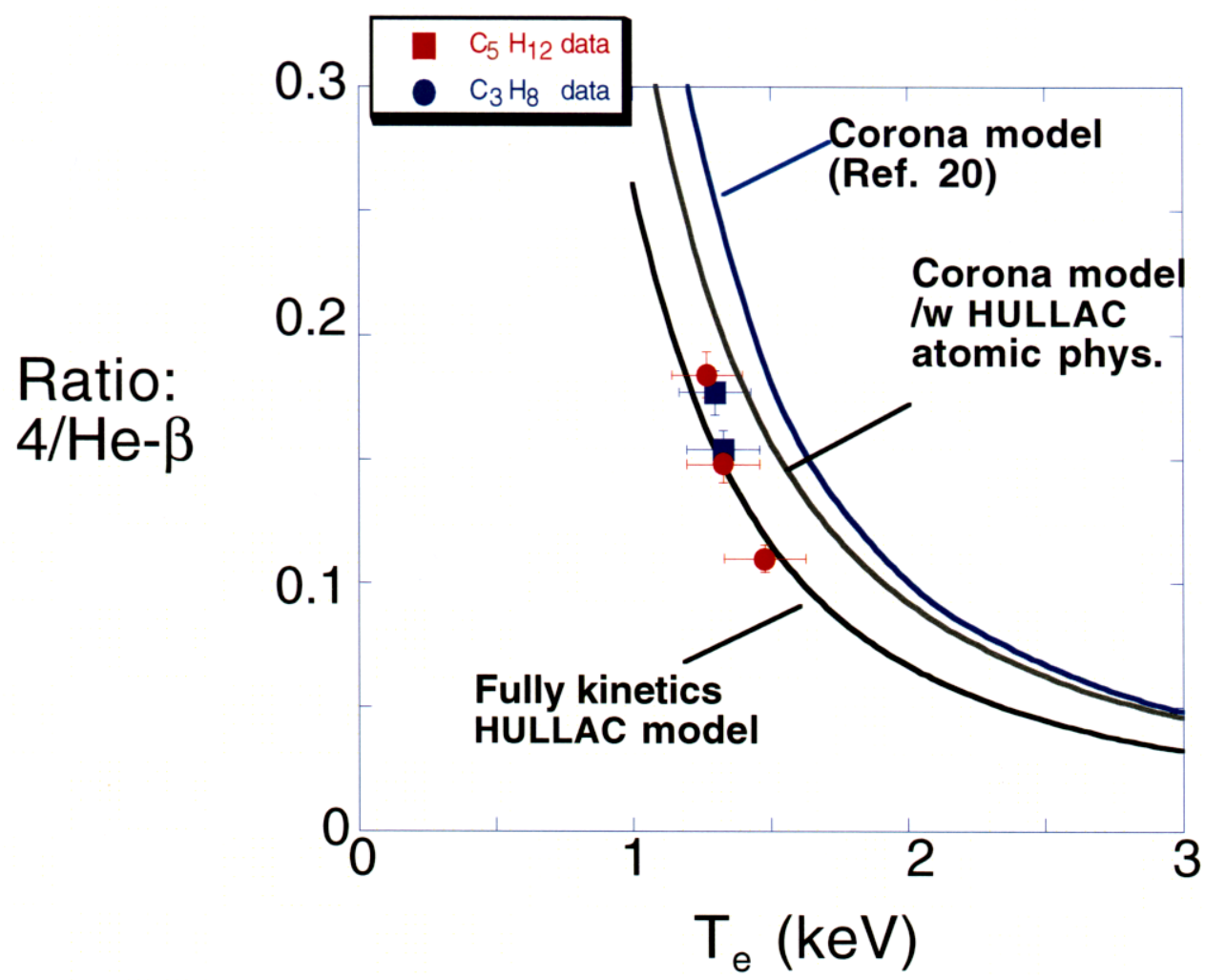

Figure 6. Comparison between the experimental line ratios from $\mathrm{C}_{3} \mathrm{H}_{8}$-filled $\left(\mathrm{n}_{\mathrm{e}}=6 \mathrm{x}\right.$ $\left.10^{20} \mathrm{~cm}^{-3}\right)$ and $\mathrm{C}_{5} \mathrm{H}_{12}$-filled $\left(\mathrm{n}_{\mathrm{e}}=1.1 \times 10^{21} \mathrm{~cm}^{-3}\right)$ gas bags with steady-state kinetics (collisional-radiative) modeling. The intensity ratio of the $\mathrm{He}-\beta$ transition (resonance plus intercombination line) to satellite feature 4 , which upper state is populated by dielectronic capture, shows excellent agreement with the fully kinetics modeling.

electron labeled 1 through 4 (4,20). For a quantitative comparison between the experiment and the spectra modeling, we fit the experimental data with a multiGaussian profile using a least-squares method (Fig.5). We obtain an estimate for the error bar of the experimental intensities of the various spectral lines by varying the fit within the noise of the data. It is in the range of $5 \%$ for the intense transitions (e.g., feature 4) to $20 \%$ for the weak transitions (e.g., feature 1). In Figure 5, the $\mathrm{n}=3$ dielectronic satellites at $0.3381 \mathrm{~nm}$ have not been included in the fit since we do not compare it to modeling. However, we checked that the $n=3$ feature as well as $n=4$ satellites $(11,20)$ do not influence the intensity of the $\mathrm{He}-\beta$ line for our conditions.

Figure 6 compares the measured intensity ratio with the results of the HULLAC calculations. It shows the ratio of the dielectronic capture satellite (labeled 4) to the $\mathrm{He}-\beta$ transition as function of the measured electron temperature for the two different electron densities. The dielectronic capture satellite

$$
1 s^{2}+e^{-} \rightarrow 1 s 2 \ell 3 \ell
$$

is primarily populated by collisional excitation from the He-like ground state and simultaneous capture of a fee electron into an excited bound state. This process is 
known to be sensitive to the electron temperature, and kinetics modeling shows no dependence on electron density for the density range of this study. We find that the experimental data are in excellent agreement with the kinetics modeling if the fully collisional-radiative HULLAC model is used.

The HULLAC calculations (15) include all singly and doubly excited energy levels with principal quantum number $n \leq 5$. The code generates atomic wavefunctions using a fully relativistic, parametric potential method that calculates the multi-configuration, intermediate coupled level energies and radiative transition rates, $A$. In addition, the code also computes semi-relativistic autoionization transition rates to the ground and excited levels of an adjacent ion. The electronimpact excitation rates between all levels of each charge state mentioned above are calculated in the distorted wave approximation. The ionic transition rates include the autoionization rates from the $\mathrm{Li}$ - to $\mathrm{He}$-like and $\mathrm{He}$-like to $\mathrm{H}$-like ions, as well as direct, impact ionization and radiative recombination rate coefficients. Radiative recombination from and collisional ionization to the bare nucleus $\mathrm{Ar}^{18+}$ is also included. These rates are used to construct the collisional-radiative rate matrix. The inverse of each ionization process, namely dielectronic recombination and threebody recombination have been found according to the principle of detailed balance. The relative populations of the four charge states and the population, $N$, in each level of each ion are then found in steady state.

From these calculations we obtain the intensity of the spectral line emission and thus the intensity ratios shown in Fig. 5 and Fig. 6. In general, for optically thin plasmas, the intensity of a radiative transition, $I_{u,}$, from the upper level, $u$, to the lower level, $\ell$, is given by the integral of the emission coefficient, $\varepsilon$, over the plasma path length:

$$
I_{u \ell}(v)=\int \varepsilon_{u \ell}(v, x) d x
$$

where $\varepsilon$ is given by the atomic transition probability, $A_{u}$ the population density of the upper atomic state, $N_{u}$, and a line shape function, $S$, that is normalized to one if integrated over the whole line profile in frequency space.

$$
\varepsilon_{u \ell}(v, x)=\frac{h v}{4 \pi} A_{u \ell} N_{u}(x) S(v, x)
$$

The comparison shows that the HULLAC calculations agree on average within $6 \%$ with the experimental intensity of the dielectronic capture satellite. Also shown in Fig. 6 are two simplified calculations using a corona approximation, i.e., for low densities the population of the upper states is determined by electron collisional excitation from the ground state, $N_{g}$, and de-excitation by radiative transitions.

$$
N_{u}=\frac{N_{g} n_{e}\langle\sigma v\rangle}{\sum A_{u \ell}}
$$

These two simplified models $(15,20)$, that use slightly different estimates for $A_{u \ell}$ and the averaged cross section for electron collisional excitation, $\langle\sigma\rangle\rangle$, overestimate the experimental ratio by $12 \%-22 \%$. This comparison indicates that a fully kinetics calculation is required to model these spectra. We observe good agreement between the experimental dielectronic capture satellite intensities and the fully kinetics model 
indicating that the populations of atomic states within one ionization stage are well understood.

On the other hand, we find that inner-shell collisional excited satellite lines are not as well modeled. These satellites are excited by collisions of free electrons with ions in the lithium-like ionization state:

$$
1 s^{2} 2 \ell+e^{-} \rightarrow 1 s 2 \ell 3 \ell^{\prime}+e^{-}
$$

Figure 7 shows the ratio of the inner-shell excited satellite feature 1 with the fully collisional-radiative HULLAC kinetics modeling.

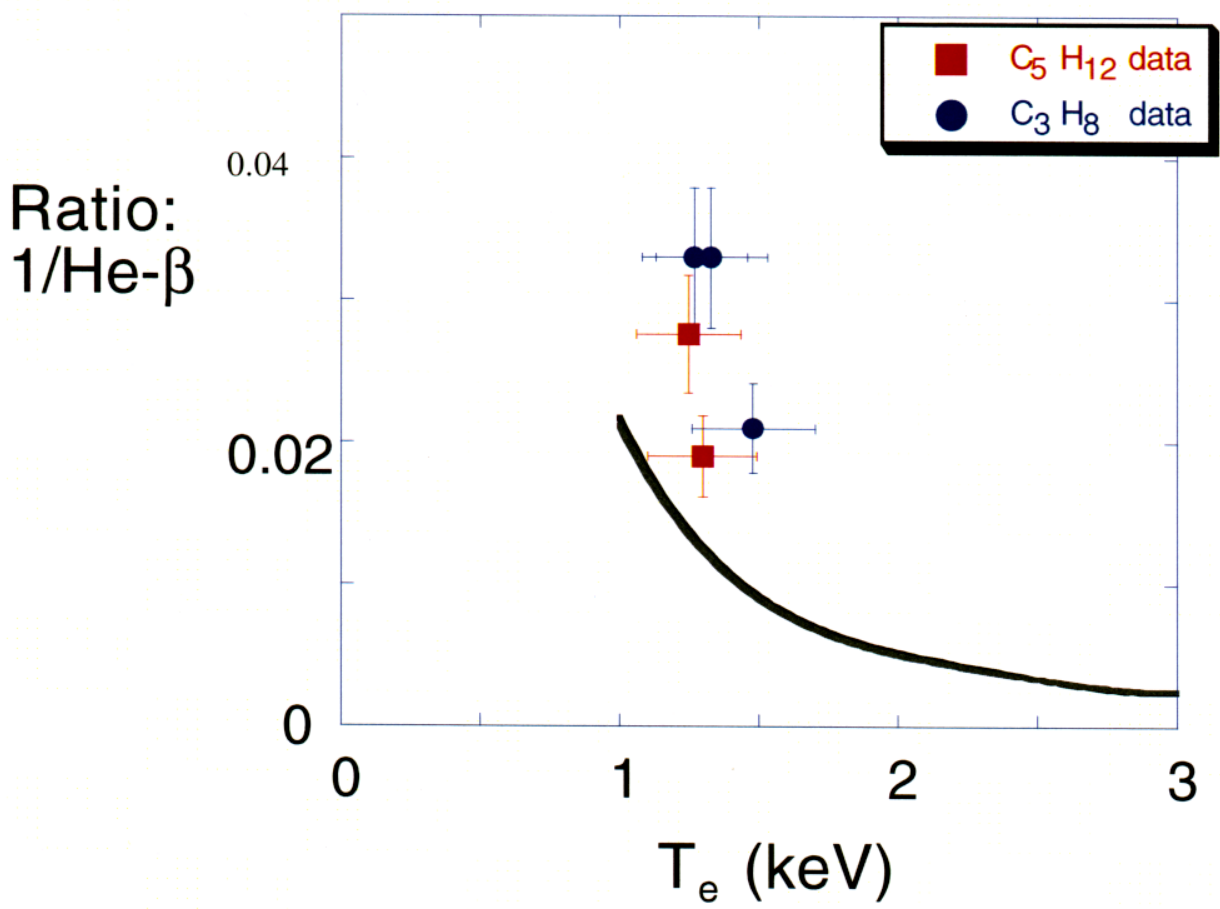

Figure 7. Comparison between the experimental line ratios from gas bags with steadystate kinetics (collisional-radiative) model calculations. The intensity ratio between the He- $\beta$ transition (resonance plus intercombination line) to satellite feature 1 , which upper state is populated by inner-shell collisional excitation, shows discrepancies of up to a factor of two compared the fully kinetics modeling.

In this case, we observe discrepancies between the data and the modeling of up to a factor of two. To ascertain the potential effects of the hot electrons and non steady state populations, we use time-dependent calculations to model the experimental conditions described above. Only for times $t<0.1 \mathrm{~ns}$, when there is a significant fraction of Li-like ions in the plasma, these calculations show that hot electrons increase the collisional excitation and therefore the intensity of the $\mathrm{He}-\beta$ transition as well as the inner-shell collisional excited satellites. However, at later times when the spectra have been measured, i.e. $0.3 \mathrm{~ns}<\mathrm{t}<0.5 \mathrm{~ns}$, the effect of the hot electrons is found to be negligible because He-like ions begin to dominate the charge state distribution so that thermal electrons dominate the collisional excitation 
process (e.g. Refs. (21)). The same time-dependent calculations further show that deviations from steady state are small for the gas bag plasma conditions and can not account for the discrepancy observed in Fig. 7.

The most likely explanation for the observed discrepancies arises from differences in the ion balance between model and experiment. The calculations indicate that at the time of the measurements about $80 \%$ of the argon ions are in the He-like ionization state while only $1 \%-2 \%$ of the ions remain in the Li-like state. Small errors in the calculation of the absolute number of Li-like ions can therefore result in large errors in the ratio of collisional excited satellites (e.g. feature 1) to the resonance transition (the He- $\beta$ transition).

\section{CONCLUSIONS}

We have performed $\mathrm{x}$-ray spectroscopic experiments in homogeneous gas bag plasmas where we independently measure the temperature with Thomson scattering. We find that collisional radiative (kinetics) modeling of the intensities of the $\mathrm{He}-\beta$ line and its dielectronic capture satellites is generally in agreement with the measured spectra. On the other hand, for the particular case of satellites arising from innershell electron collisional excitation, we find discrepancies of up to a factor of two between experiment and kinetics models. We have ruled out possible effects on the line emission due to plasma gradients, radiative transport, and suprathermal electron excitation leaving errors in the atomic physics modeling to be the most likely explanation.

The determination that there are problems with the collisionally populated states is important for the interpretation of inertial confinement fusion capsule implosions where electron densities and temperature have been measured using the spectral line shape of the He- $\beta$ transition of Ar XVII. The analysis of the implosion data has required Stark broadening calculations coupled to a kinetics model to calculate the detailed line intensities and widths. Despite remaining discrepancies, the good agreement between the experimental dielectronic capture satellites and the HULLAC calculations suggests that HULLAC is a more appropriate code for the construction of the kinetics models of the He- $\beta$ complex from high density plasmas than previously used codes (e.g. MCDF). HULLAC results in higher temperatures for the implosion conditions of $\operatorname{Ref}(5,6)$ in closer agreement with the $2-\mathrm{D}$ radiation hydrodynamic modeling and other spectroscopic techniques. These results indicate that benchmarking kinetics codes with Thomson scattering is an important area in present ICF research.

\section{ACKNOWLEDGMENTS}

We would like to thank A. L. Osterheld, C. Decker, L. Lours, and P. E. Young for helpful discussions. This work was performed under the auspices of the U.S. Department of Energy by University of California Lawrence Livermore National Laboratory under contract No. W-7405-ENG-48. 


\section{REFERENCES}

1. B. J. MacGowan et al., Phys. Plasmas 3, 2029, (1996).

2. S. H. Glenzer, C. A. Back, K. G. Estabrook, B. J. MacGowan, D. S. Montgomery, R. K. Kirkwood, J. D. Moody, D. H. Munro, G. F. Stone, Phys. Rev. E 55, 927 (1997).

3. P. G. Burkhalter, J. Shiloh, A. Fisher, and R. D. Cowan, J. Appl. Phys. 50, 4532 (1979).

4. V. A. Boiko, S. A. Pikuz, U. I. Safronova, and A. Ya. Faenov, Mont. Not. R. Astr. Soc. 185, 789 (1978).

5. B. A. Hammel, P. Bell, C. J. Keane, R. W. Lee, and C. L. S. Lewis, Rev. Sci. Instrum. 61, 2774 (1990). B. A. Hammel, C. J. Keane, M. D. Cable, D. R. Kania, J. D. Kilkenny, R. W. Lee, and R. Pasha, Phys. Rev. Lett. 70, 1263 (1993). B. A. Hammel, C. J. Keane, T. R. Dittrich, D. R. Kania, J. D. Kilkenny, R. W. Lee, and W. K. Levedahl, J. Quant. Spectrosc. Radiat. Transfer 51, 113 (1994).

6. N. C. Woolsey, B. A. Hammel, C. J. Keane, A. Asfaw, C. A. Back, J. C. Moreno, J. K. Nash, A. Calisti, C. Mosse, R. Stamm, B. Talin, L. S. Klein, and R. W. Lee, Phys. Rev. E 56, 2314 (1997). ibid, Phys. Rev. E 57, 4650 (1998). N. C. Woolsey et al., Phys. Rev. E 53, 3696 (1996). \{lit ibid\}, J. Quant. Spectrosc. Radiat. Transfer 58, 975 (1997).

7. M. D. Cable, S. P. Hatchett, J. A. Caird, J. D. Kilkenny, H. N. Kornblum, S. M. Lane, C. Laumann, R. A. Lerche, T. J. Murphy, J. Murray, M. B. Nelson, D. W. Phillion, H. Powell, and D. B. Ress, Phys. Rev. Lett. 73, 2316 (1994).

8. A. H. Gabriel, Mon. Not. R. Astron. Soc. 160, 99 (1972).

9. R. W. Lee, B. L. Whitten, R. E. Strout, II, J. Quant. Spectrosc. Radiat. Transfer 32, 91 (1984).

10. C. F. Hooper, Jr., D. P. Kilcrease, R. C. Mancini, L. A. Woltz, D. K. Bradley, P. A. Jaanimagi, and M. C. Richardson, Phys. Rev. Lett. 63, 1267 (1989). C. F. Hooper et al., Quant. Spectrosc. Radiat. Transfer 44, 79 (1990).

11. R. C. Mancini, C. F. Hooper, N. D. Delamater, A. Hauer, C. J. Keane, B. A. Hammel, J. K. Nash, Rev. Sci. Instrum. 63, 5119 (1992). D. A. Haynes, D. T. Garber, C. F. Hooper, R. C. Mancini, Y. T. Lee, D. K. Bradley, J. Delettrez, R. Epstein, P. A. Jaanimagi, Phys. Rev. E 53, 1042 (1996). I. E. Golovkin and R. C. Mancini, J. Quant. Spectrosc. Radiat. Transfer 65, $273(2000)$.

12. H.-J. Kunze, in Plasma Diagnostics, edited by W. Lochte-Holtgreven (North-Holland, Amsterdam, 1968) p.550.

13. S. H. Glenzer, W. E. Alley, K. G. Estabrook, J. D. De Groot, M. Haines, I. H. Hammer, J.P. Jaddaud, B. J. MacGowan, J. D. Moody, W. Rozmus, L. J. Suter, T. L. Weiland, and E. A. Williams, Phys. Plasmas 6, 2117 (1999). S. H. Glenzer et al, Rev. Sci. Intrum. 70 1089 (1999). S. H. Glenzer et al., Phys. Rev. Lett. 8297 (1999).

14. G. Zimmerman and W. Kruer, Comments Plasma Phys. Controlled Fusion 2, 85 (1975).

15. A. Bar-Shalom and M. Klapisch, Computer Phys. Comm. 50, 375 (1988). M. Klapisch, Computer Phys. Comm. 2, 239 (1971). M. Klapisch, J. Schwob, B. Fraenkel, and J. Oreg, J. Opt. Soc. Am. 67, 148 (1977). J. Oreg, W. H. Goldstein, M. Klapisch, and A. BarShalom, Phys. Rev. A 44, 1750 (1991). A. Bar-Shalom, M. Klapisch, and J. Oreg, Phys. Rev. A 38, 1773 (1988).

16. E. M. Campbell, J. T. Hunt, E. S. Bliss, D. R. Speck, and R. P. Drake, Rev. Sci. Intrum. 57, 2101 (1986).

17. C. A. Back, R. L. Kauffman, P. M. Bell, and J. D. Kilkenny, Rev. Sci. Instrum. 66, 764 (1995).

18. J. D. Kilkenny, Laser Part. Beams 9, 49 (1991).

19. J. A. Fejer, Can. J. Phys. 39, 716 (1961). D. E. Evans, Plasma Phys. 12, 573 (1970). S. H. Glenzer, C. A. Back, K. G. Estabrook, R. Wallace, K. Baker, B. J. MacGowan, B. A. Hammel, R. E. Cid and J. S. De Groot, Phys. Rev. Lett. 77, 1496 (1996).

20. P. Beiersdorfer, A. L. Osterheld, T. W. Phillips, M. Bitter, K. W. Hill, and S. von Goeler, Phys. Rev. E 52, 1980 (1995).

21. F. B. Rosmej, J. Phys. B 28, L747 (1995). F. B. Rosmej, J. Quant. Spectrosc. Radiat. Transfer 51, 319 (1994). 